\title{
Strates
}

STRATES Matériaux pour la recherche en sciences sociales

13 | 2007

Paysage urbain: genèse, représentations, enjeux contemporains

\section{Paysage urbain: d'une thématique à un objet de recherche}

Hélène Jannière et Frédéric Pousin

\section{OpenEdition}

Journals

Édition électronique

URL : http://journals.openedition.org/strates/4953

ISSN : $1777-5442$

Éditeur

Laboratoire Ladyss

Édition imprimée

Date de publication : 31 décembre 2007

ISSN : 0768-8067

Référence électronique

Hélène Jannière et Frédéric Pousin, « Paysage urbain: d'une thématique à un objet de recherche », Strates [En ligne], 13 | 2007, mis en ligne le 22 octobre 2008, consulté le 07 septembre 2020. URL http://journals.openedition.org/strates/4953

Ce document a été généré automatiquement le 7 septembre 2020

Tous droits réservés 


\title{
Paysage urbain: d'une thématique à un objet de recherche
}

\author{
Hélène Jannière et Frédéric Pousin
}

1 Le volume présenté à l'attention du lecteur est issu d'un séminaire, tenu de 2003 à 2006 à l'Université de Paris-I, qui s'était donné pour objectif d'interroger une notion, «le paysage urbain », dont les usages contradictoires et l'actualité grandissante méritent qu'on s'attache à la mieux cerner. Il nous a semblé stratégique de faire saillir la multiplicité de ces usages, selon des perspectives disciplinaires, professionnelles, artistiques différentes. En effet, pour les géographes, la catégorie du paysage a toute légitimité dès lors qu'elle renvoie au rural. Elle apparaît plus discutable appliquée à l'urbain et rencontre alors de manière étroite l'urbanisme. Si en architecture et en urbanisme, la notion de paysage urbain est loin d'être absente, elle ne participe toutefois pas des catégories opératoires canoniques ${ }^{1}$. On tendrait alors à se tourner vers les paysagistes pour en saisir le caractère opératoire, mais en vain, car elle s'avère ici polémique. En revanche en photographie, de nombreuses démarches lui sont dédiées². Telles sont les raisons pour lesquelles nous avons convié à s'exprimer des intervenants représentant des champs différents, afin de croiser leurs regards tant sur cette notion de paysage urbain que sur les usages qui en sont faits.

2 En outre, ce séminaire est venu prolonger un programme de recherche, ouvert de longue date, centré sur la question du paysage urbain dans le champ de l'architecture et du paysagisme ${ }^{3}$. Conçu comme une ouverture vers la discipline Géographie et vers les pratiques artistiques à travers la photographie, il visait à faire converger les travaux menés sur le sujet.

3 Il s'est trouvé que ce séminaire a rencontré une certaine actualité éditoriale, puisque, dans le même temps, plusieurs ouvrages - investigations théoriques ou ouvrages plus directement destinés aux professionnels de l'aménagement ${ }^{4}$ - étaient consacrés totalement ou partiellement à cette question.

4 Le présent volume est fragmentaire : il ne rassemble qu'une partie des contributions au séminaire $^{5}$. Mais la publication des premiers résultats dans une collection intitulée Matériaux pour la recherche s'avère tout à fait adéquate. Une telle publication, parce 
qu'elle permet de tracer des directions de recherche pour baliser un domaine, autant que de faire se rencontrer des éléments de réflexion, représente un jalon que nous espérons marquant dans un chantier qui reste largement ouvert.

Actualité de l'expression paysage urbain

5 Nous l'avons dit, ce volume paraît à un moment où la problématique du paysage et de l'urbain est au cœur de plusieurs livraisons, que nous voudrions caractériser brièvement afin d'expliciter l'originalité de notre démarche.

6 Un premier ensemble d'ouvrages part d'une réflexion générale sur le paysage pour interroger l'urbain dans ce qu'il représente de particulier. Ainsi, le livre collectif Paysages en perspectives ${ }^{6}$ propose un état des lieux des enjeux pluriels que pose le paysage, en soulignant l'évolution des réflexions dans le domaine des paysages en milieu urbain. Les auteurs constatent le renouveau qui se fait jour dans les pratiques aménagistes, consistant à doter l'infrastructure d'un potentiel de qualification positive des territoires. En outre, ils constatent, jusqu'à une période très récente, une certaine occultation du paysage urbain dans l'aménagement, qu'ils attribuent à une inadéquation entre le caractère unitaire dont la notion serait porteuse et le caractère hétérogène et dynamique des conditions urbaines contemporaines. Le chapitre consacré aux paysages de l'urbain, expression préférée par les auteurs à paysage urbain, recense à travers les discours artistiques, savants ou professionnels, les différents usages du terme de paysage tels qu'ils s'appliquent au contexte particulier de la ville et des territoires urbains. Il s'efforce ensuite de dégager les attributs explicites ou implicites associés à de tels usages, en termes de formes, de thèmes ou d'intentions, catégories qui recoupent les différentes finalités discursives repérées : représentation, interprétation, projet. Les auteurs en concluent que la notion de paysage en milieu urbain implique « une qualification des formes du territoire de la ville par le regard, et résulte d'une distanciation plurielle complexe ${ }^{7} »$.

7 Adoptant une démarche beaucoup moins conceptuelle, Michael Spens, dans Paysages contemporains $^{8}$, examine les créations récentes des paysagistes de différentes générations ${ }^{9}$ pour constater qu'outre les programmes traditionnels de parcs et jardins, une part importante des réalisations est consacrée aux interventions urbaines. L'auteur y insiste sur l'actualité de l'idée d'espaces interstitiels dans nos villes, à la croisée de trames urbaines diversifiées, qui offrent des opportunités de réalisations génératrices d'identité pour nos paysages urbains contemporains. Il mentionne également l'apport des praticiens qui ont su reconnaître le rôle du paysage, qu'il soit minéral ou végétal, dans l'intervention urbaine, permettant selon lui d'en renouveler ainsi les fondements.

8 L'expérience esthétique est également convoquée à l'endroit de l'expérience paysagère. Liant la compréhension du paysage à celle de l'ornement, l'ouvrage Paysage et ornement $^{10}$ dirigé par Didier Laroque et Baldine Saint-Girons tente de prolonger et renouveler la réflexion de la décennie 1990 qui, en France, avait tenté de fonder théoriquement la réflexion pluridisciplinaire sur le paysage. Anne Léon-Miehe y défend l'idée que, saisi qu'il est entre fiction et description, le paysage est avant tout produit par le langage, récusant ainsi le rapport à la peinture comme principe même du paysage ${ }^{11}$. Pas plus que le tableau n'est constitutif du paysage, la vue n'en est un sens privilégié, et ce sont bien tous les sens qui sont à l'œuvre pour composer le paysage. De même Philippe Poullaouec considère-t-il ailleurs que l'exploration de l'expérience plurisensorielle du paysage, en territoires urbains ou naturels, constitue un axe de recherche particulièrement porteur. 
9 Un second ensemble d'ouvrages part de la matérialité urbaine pour interroger le paysage. Ainsi, c'est à partir des infrastructures de transport que Marc Desportes dans Paysage en mouvement ${ }^{12}$ croise histoire des techniques, histoire culturelle et théorie du paysage. Il interroge l'espace dans lequel s'inscrivent les ouvrages techniques, les représentations spatiales tant de leurs concepteurs que de leurs utilisateurs. Chaque grande technique de transport recèle un paysage, en ce qu'elle modèle une approche de l'espace traversé, comme il le montre dans ses études du paysage de la route au XVIII siècle, du chemin de fer au xix ${ }^{e}$ siècle, des premières automobiles, de l'autoroute au $\mathrm{xx}^{\mathrm{e}}$ siècle, et du paysage vu d'avion. Ainsi se définissent des paysages de la technique, caractérisés par les regards induits par les infrastructures.

Dans Méandres, penser le paysage urbain ${ }^{13}$, Peter Versteegh fait le constat d'une urbanisation généralisée et interroge le paysage comme voie originale pour l'aménagement. Comment penser l'espace des grandes métropoles, des conurbations et autres formations urbaines multipolaires qui structurent aujourd'hui nos territoires? Au-delà des qualificatifs usités : discontinu, fragmentaire, organisation chaotique, il y aurait pour P. Versteegh un défi à décrire la ville comme un paysage urbain. Cet ouvrage collectif vise à structurer une réflexion sur le paysage contemporain, autour d'une hypothèse centrale : ne plus partir des modèles mais plutôt de la réalité de la production contemporaine, mêlant un complexe de tissu urbain et de fragments paysagers en mouvance. Il y a là une inversion conceptuelle qui s'avère nécessaire aux yeux de P.Versteegh qui propose diverses pistes pour la mettre en œuvre parmi lesquelles, la description photographique.

11 Nous ne sommes pas revenus dans ce volume sur les origines littéraires et picturales du paysage urbain, domaine fort exploré par ailleurs. Selon Françoise Chenet-Faugeras, l'expression paysage urbain a été utilisée par les écrivains et les poètes à la fin du XIX ${ }^{e}$ siècle pour décrire les formes de la ville offrant de nouvelles sensations, et l'«observation décontractée de la vie urbaine » dans les métropoles. Apparue dans la littérature au moment de la transformation radicale des villes, cette expression marquerait ainsi le passage d'une totalité finie et cernable, évoquée par l'expression "paysage de la ville", à une ville fragmentée, "débordée par ses banlieues": le paysage urbain « correspond au passage insensible d'une forme aux contours définis et stables à l'informe de l'agglomération urbaine ${ }^{14} »$. Dans une autre acception, les historiens d'art au début $d u \mathrm{xx}^{\mathrm{e}}$ siècle évoquent le paysage urbain en tant que genre pictural.

12 En 2005, la revue Eidôlon a fait le point sur le paysage urbain de 1830 à nos jours ${ }^{15}$. Bien que revendiquant une approche pluridisciplinaire, ce volume se consacre presque entièrement à la littérature, hormis un nombre réduit d'articles sur le cinéma dans les années 1920, la publicité et la représentation des paysages industriels dans la peinture.

13 Les transformations urbaines advenues au $\mathrm{XIX}^{\mathrm{e}}$ siècle ${ }^{16}$ et au début du XX⿳亠丷厂 sont pour les auteurs déterminantes dans la formation d'un regard sur ce nouveau paysage urbain. Cette livraison interroge particulièrement le changement de regard des artistes, la nécessaire adaptation des catégories esthétiques héritées de la représentation du paysage naturel sur cette réalité urbaine: les auteurs font ainsi l'hypothèse que les représentations du paysage urbain découlent de celles du paysage naturel. «Comment et à travers quelles difficultés se met en place une nouvelle écriture du paysage urbain (esthétisation, anthropomorphisation, détournements divers) ${ }^{17}$ ? » L'objectif du volume réuni par Gérard Peylet et Peter Kuon est de montrer qu'à l'époque industrielle et de la 
modernité artistique, un nouveau rapport s'est instauré entre le sujet (le regard) et l'objet (le paysage), ce rapport étant selon eux constitutif du paysage urbain. Mais malgré cette hypothèse, les auteurs souhaitent confronter - et de la sorte implicitement opposer - le "paysage urbain réel» (l'architecture et la ville) avec le paysage urbain entendu comme perception, afin d'étudier la «relation de la représentation au référent ${ }^{18} »$.

Construction d'un objet

C'est notamment une telle disjonction entre " paysage réel » et "paysage perçu » que nous souhaiterions mettre en question dans le présent volume: notre hypothèse est que géographes, architectes et urbanistes interprètent par leurs analyses ou modifient par leurs pratiques professionnelles un "paysage urbain» qui n'en est pas moins " paysage réel ».

Notre stratégie n'a donc pas été de partir d'une prétendue réalité, mais bien plutôt des disciplines et des pratiques qui font travailler la notion de paysage urbain afin de mieux comprendre en quoi celle-ci participe de constructions scientifiques et/ou professionnelles qu'elle contribue d'ailleurs à faire bouger. Ainsi, selon nous, les enjeux portés par cette notion peuvent-ils être méthodiquement sériés. Plusieurs colloques ou ouvrages collectifs, tant sur le paysage ${ }^{19}$ que sur le paysage urbain examinent en effet l'objet " paysage urbain » à travers un très vaste spectre de pratiques ou de disciplines (du cinéma, de la publicité à la musique, dans la littérature et l'histoire de la peinture...), sans pouvoir toujours en cerner avec suffisamment de précision les différentes acceptions d'un domaine à l'autre. À l'inverse, le séminaire dont est issu ce numéro de Strates a tenté de construire l'objet paysage urbain par une exploration dans trois directions : une perspective épistémologique et historique, engageant plusieurs enquêtes sur la généalogie de la notion, une exploration de ses enjeux professionnels et enfin une réflexion sur ses enjeux esthétiques.

Notre parti s'affirme par conséquent comme ouvertement épistémologique, en ce qu'il interroge une notion dans un contexte interdisciplinaire et pluriprofessionnel. Une difficulté persiste toutefois, qui consiste à accorder toute son importance au contexte culturel au sein duquel s'élabore la signification d'une telle notion. Déjà par le passé, nous avions ancré notre enquête dans des terrains et des temporalités où cette notion était la plus saillante, en Grande-Bretagne dans les années 1940 à $1960^{20}$. Lors du séminaire, nous avons choisi de poursuivre notre effort sur la diffusion de la notion et la multiplicité des terrains, ce qui appelle quelques précisions méthodologiques. Il apparaît auparavant essentiel d'exposer ce qui fut pour cette notion un moment privilégié, un point d'émergence, incontournable pour la réflexion qui est la nôtre, à savoir le townscape.

Le townscape

17 Comme en France, le terme de paysage urbain apparaît en Angleterre à la fin du XIX siècle, dans les domaines de la littérature et de la critique d'art. Il y qualifie le charme désuet des villes historiques, en particulier des villes préindustrielles. Pour cette raison, se sont développées sous cette appellation des méthodes de conservation des quartiers historiques et de l'architecture urbaine. Cette connotation protectionniste n'est toutefois pas la seule. Le terme de townscape a été fortement réinvesti à la fin des années 1940, en Angleterre, dans les domaines de l'architecture et de la planification urbaine $^{21}$. En 1948, Thomas Sharp ${ }^{22}$ utilise le terme pour dénommer une pratique de 
restructuration des villes (improving the cities) par analogie avec l'art des paysagistes et grands propriétaires terriens qui restructurèrent leurs domaines au $\mathrm{XVIII}^{\mathrm{e}}$ siècle.

Si l'ancrage dans la grande tradition anglaise du pittoresque est indéniable, le townscape en tant que doctrine urbanistique, telle qu'elle fut forgée par Hugh de Cronin Hastings et Gordon Cullen, se veut avant tout une nouvelle tradition architecturale, en rupture avec celle du Mouvement moderne ${ }^{23}$. Cette nouvelle tradition se définit par une appréhension globale de l'environnement et la primauté accordée à sa dimension visuelle. La perception et le mouvement sont au centre de la doctrine et des méthodes promues par ces deux auteurs. L'hypothèse alors développée au nom du townscape était que la restructuration urbaine pouvait être accomplie d'une manière objective grâce à la compréhension des effets produits par la mise en relation des éléments physiques de l'environnement (un art de l'ensemble). Les théories de la perception conféraient à cette approche son soubassement philosophique (phénoménologie) et scientifique (psychologie expérimentale).

19 Afin de rendre compte d'une telle approche globale de l'environnement, plusieurs thématiques peuvent être identifiées, tour à tour développées par la revue anglaise The Architectural Review : l'unicité de lieu [genius loci], la critique de la dispersion urbaine, l'extension de la notion de conservation à la préservation de groupes de bâtiments ou d'espaces importants pour la forme urbaine, la revendication de fondements savants ou populaires pour la conception raisonnée du paysage, enfin la dimension dramatique du paysage urbain en tant que réponse émotionnelle à l'environnement physique.

20 Au titre des applications auxquelles ont donné lieu le mouvement du townscape, on retiendra au premier chef l'aménagement d'espaces publics, puis des projets pour les rives de la Tamise, enfin des projets de villes nouvelles régionales ${ }^{24}$ (1964-1965). Par ailleurs, le townscape a continué de rencontrer les tendances protectionnistes donnant lieu à des démarches de conservation patrimoniale.

21 En outre, le townscape a pu être vu aussi comme une "philosophie de l'urbanisme" (Urban Design), dans la mesure où il suppose une connaissance du comportement humain en ville. G. Cullen parle d'une appropriation de l'espace qui donne sa place à la série des besoins humains et des émotions, mais également à la circulation et au mouvement qui contribuent à rendre vivants les espaces de la ville. Les intuitions de G. Cullen sur les ressorts du visuel et les besoins humains qui s'y rapportent ont trouvé leur validation dans les travaux postérieurs de psychologie environnementale et d'anthropologie.

Partant de la réflexion menée sur le townscape, il nous est apparu possible d'engager la poursuite du travail selon les trois directions de recherche que nous allons maintenant présenter.

Une perspective diachronique et épistémologique

La thématique du paysage urbain est à la croisée de plusieurs domaines. Au-delà de l'architecture et de l'urbanisme, nous avons choisi d'explorer les liens existants avec la géographie comme autre domaine disciplinaire. Puis le domaine du paysagisme vient ici compléter le champ opérationnel.

24 L'enquête effectuée dans la littérature professionnelle par Hélène Jannière s'est attachée à suivre, en France, l'apparition du terme dans les manuels et dans la presse professionnelle d'urbanisme. Cette enquête a rencontré des questions d'ordre méthodologique relatives à la distinction terme/notion ; la locution " paysage urbain " 
n'étant pas la seule manifestation possible de la notion, notamment dans l'entre-deuxguerres. La périodisation proposée permet d'appréhender dans la diachronie les transformations qui touchent un terme, le dotent de nouvelles valeurs: opératoires, descriptives ou critiques. Grâce à cette dimension, il est possible d'accéder à l'épaisseur du terme comme de la notion.

Une plongée dans la littérature de la géographie urbaine a été menée parallèlement. L'article de Xavier Michel s'est concentré sur la période 1960-1970, période durant laquelle le vocable est utilisé largement par les géographes. La lecture minutieuse des ouvrages, des manuels et des thèses qui étayent son enquête ouvre des perspectives de dialogue avec l'article d'Hélène Jannière. Il est d'ailleurs significatif que chacun ait lancé des ponts vers le domaine de l'autre. La contribution de Frédéric Pousin ouvre à nouveau le dossier anglais, pour interroger cette fois les procédés éditoriaux élaborés pour susciter un débat public qui sera implicitement ou explicitement revendiqué au moment où le townscape sera institué comme référence par les urbanistes et paysagistes français. Ce sont principalement les procédés rhétoriques et visuels qui servent de modèle aux publications françaises. Enfin, l'enquête menée par Florent Hébert interroge les discours des paysagistes sur l'urbain, qu'il éclaire au travers des modèles culturels du paysage, de la demande sociale de nature, ou de la catégorie professionnelle canonique de site. Dans les diverses formes de représentation culturelles, sociales mais aussi les représentations iconiques qui assurent le transfert des discours aux actes, c'est la dimension opératoire de la notion qui est ici travaillée.

Enjeux esthétiques

Nous avons choisi d'interroger l'esthétique urbaine à partir de ce qu'en disent les disciplines que nous observons, aussi bien que la réflexion esthétique elle-même.

Dans un petit livre stimulant, Adieu à l'esthétique ${ }^{25}$, Jean-Marie Schaeffer tire les conséquences des débats qui se sont déroulés tout au long des années 1990 autour des œuvres d'art et de l'idée de relation esthétique. Ces débats ont porté, non sur l'esthétique en tant que discipline philosophique, mais sur l'expérience esthétique, la relation esthétique, ou encore la conduite esthétique. Aux yeux des chercheurs qui explorent les relations qui se tissent entre paysage et esthétique, ce sont les significations découlant des expériences faites par les individus qui participent à transformer certains milieux ou territoires en paysages, comme le montre Sophie Bonin ici même.

Un tel recentrement sur l'expérience comme ressort de la construction esthétique conduit également à une approche «désacralisée » de la production artistique. C'est dans cette perspective que la pratique photographique est ici interrogée. Quelle forme de regard sur l'urbain la photographie produit-elle, quelle expérience exprime-t-elle et quelle est son efficace en tant que forme de représentation?

Mark Dorrian choisit d'interroger la structure de la vision à travers la vue d'en haut. Il lui a fallu définir les limites d'une telle thématique (aerial vue). À partir de la vue d'en haut, qui toujours est déjà en relation avec la position verticale de l'être humain, il soulève une série de questions relatives à la proximité et aux échelles. Il distingue ensuite trois catégories d'image pour une «vue d'en haut " statique : la vue oblique, la vue verticale et le diagramme. Les deux premières catégories sont analysées tant dans leur dimension historique que dans leurs enjeux formels. Quant au diagramme, il convient de définir à quelles conditions il relève d'une « vue d'en haut ». 


$$
\text { rapportées par les articles du Bulletin du Touring Club de Belgique. Il souligne, de ce point }
$$
de vue, les décalages entre iconographie et récit.

Enjeux professionnels et environnementaux

31 l'introduction du paysage dans le champ urbain ouvre sur des enjeux professionnels qui décloisonnent les expertises techniques et positionnent l'aménagement dans une réalité pluriprofessionnelle. Nous avons souhaité explorer ici deux terrains ainsi que deux moments historiques où se sont nouées, en France, des interactions étroites entre urbanisme et paysagisme : les grands ensembles et les villes nouvelles ${ }^{27}$.

La contribution de Bernadette Blanchon porte sur le traitement des espaces collectifs et des espaces verts dans les grands ensembles. Elle distingue plusieurs périodes de l'intervention paysagère, en situant les paysagistes par rapport à leur formation et par rapport à la catégorie canonique du site. Néanmoins, durant la période qu'elle analyse, la volonté d'un corps professionnel de conquérir une position dans le champ de l'urbanisme apparaît comme une constante.

33 L'intervention en ville nouvelle a nécessité de travailler sur des objets comme les réseaux viaires, sur lesquels s'affrontent tout particulièrement différentes logiques professionnelles. Viviane Claude met en évidence que des objets complexes tels que les espaces libres en ville nouvelle, souvent caractérisés par la grande échelle, ont été révélateurs de nouvelles compétences. La grande échelle des paysages de villes nouvelles a motivé l'appel aux paysagistes. Mais plus encore, chacun des acteurs aurait été amené à reconsidérer les frontières de ses propres compétences et de son expérience professionnelle antérieure en se confrontant à de tels objets.

Du côté des enjeux environnementaux, l'approche de la ville en termes de paysage signifie redonner une identité positive, une sociabilité, à des espaces ou des territoires qui en sont dépourvus. Pour Sophie Bonin, il faut distinguer l'échelle locale du paysage - où se construit le sens pour les individus - de l'échelle globale du paysage qui renvoie à l'expérience partagée. C'est donc à l'échelle locale que se pose la question du changement de regard. Son article est centré sur la relation ville-fleuve pour traiter des enjeux écologiques, relation qui s'avère un bon objet d'étude pour interroger la problématique du paysage urbain, dès lors qu'on privilégie l'échelle locale pour l'observation, sans s'y limiter pour autant.

Claire Roullet-Sureau présente le cas des vallées non domaniales à travers l'exemple de l'urbanisation de la vallée de l'orge. Si dans l'aménagement hydraulique, la mise en valeur paysagère de l'espace urbain n'est pas première, le rôle du paysage a été cependant déterminant pour déclencher la prise de conscience des dysfonctionnements. Il a permis l'implication d'acteurs qui, jusque-là, n'étaient pas convoqués dans l'aménagement.

Mais la structuration du paysage urbain en objet de recherche s'appuie également sur une autre dimension : la circulation de la notion d'un champ disciplinaire à l'autre et sa circulation géographique.

Circulation de la notion : une notion interdisciplinaire

37

L'ensemble des enquêtes sur la généalogie de la notion nous a amenés à considérer que celle-ci se construit aux frontières de plusieurs pratiques - architecture, urbanisme, paysagisme - et de plusieurs disciplines - géographie, histoire de l'art et esthétique. 
Une des hypothèses qui structurent ce volume est que la circulation d'une discipline à l'autre est constitutive de la notion de paysage urbain : c'est en effet la particularité du paysage urbain, que de ne pas être un objet légitime pour chacune des disciplines prises séparément, mais de le devenir dès lors que se met en place une logique interdisciplinaire, condition pour être constitué en objet de recherche. Au programme du séminaire, figurait l'intention d'enquêter sur les transferts notionnels entre la géographie, l'urbanisme, le paysagisme, les pratiques de conservation et de patrimoine. Les premiers résultats de cette interrogation concernent des similitudes de périodisation. Ils dégagent également des objets privilégiés sur lesquels semble se cristalliser la notion de paysage urbain. Les premiers jalons ont ainsi été posés quant aux transformations, altérations ou enrichissements de cette notion d'un champ disciplinaire à l'autre : leur analyse reste un chantier ouvert.

Géographie et urbanisme

Déjà évoquée ici, l'étroite relation entre géographie et urbanisme s'est illustrée par des similitudes entre les manuels de géographie et la littérature professionnelle des urbanistes français tout au long $\mathrm{du} \mathrm{xx}^{\mathrm{e}}$ siècle, qui révèlent des périodisations convergentes quant à l'usage de la notion de paysage urbain : notamment sa rareté avant la deuxième guerre mondiale, son éclipse dans les années d'après-guerre jusqu'aux années 1960. Ces périodisations ont constitué l'un des points de départ du séminaire, où la notion de paysage urbain a d'abord été repérée à partir de la présence de la locution, considérée comme sa manifestation la plus évidente. Nous avons continué d'explorer ces rapprochements lors de la journée d'étude organisée conjointement avec le Laboratoire de géographie sociale et d'études urbaines de l'EHESS, spécifiquement consacrée à une approche comparative entre géographie et urbanisme ${ }^{28}$. D'un point de vue épistémologique, les conférences et débats de cette journée ont soulevé la question de la distinction entre terme et notion dans ces enquêtes épistémologiques. Ils ont ainsi confirmé l'hypothèse soulevée à propos des manuels d'urbanisme de l'entre-deux-guerres. Sans nécessairement faire usage du terme, ceux-ci véhiculent la notion de paysage urbain, repérable car associée à un certain nombre de thématiques et de termes en relation avec l'esthétique urbaine, identifiés par ailleurs comme constitutifs du paysage urbain, ou comme des cooccurrences de ce terme.

Outre le fait qu'urbanistes et géographes se lisent ${ }^{29}$, la circulation des notions entre géographie et urbanisme a pu être stimulée (ce qui ouvre une piste de recherche supplémentaire) par la proximité et les interactions entre les acteurs de l'aménagement: dans les années 1960, plusieurs géographes tels que Philippe Pinchemel occupent des fonctions dans des commissions et groupes d'études au ministère de la Reconstruction et de l'Urbanisme; on peut citer également Pierre George, vice-président du Centre de recherches d'urbanisme (CRU), fondé en 1962. Or, c'est dans cette décennie qu'est réactivée en France la notion de paysage urbain. Géographes et urbanistes l'emploient à propos d'objets communs qui cristallisent la notion à la fois dans l'urbanisme et dans la géographie. On note ainsi, dans la première moitié des années 1960, la réapparition presque synchrone de la locution " paysage urbain » chez les géographes et chez les urbanistes et architectes en France, notamment dans la description des changements induits par l'apparition des grands ensembles sur le territoire français.

Histoire de l'art, patrimoine et rénovation urbaine 

littérature, la discipline où l'on repère à la fin $\mathrm{du} \mathrm{XIX}^{\mathrm{e}}$ siècle les premières occurrences du terme paysage urbain. Il existe des transferts de l'histoire de l'art à l'histoire des villes et à la géographie. En 1926, Pierre Lavedan applique ainsi la notion de paysage urbain issue de l'histoire de l'art (la discipline d'origine de cet auteur) et concernant les vues de villes, à son essai d'histoire des formes urbaines, puis, en 1959, à la seconde édition de la Géographie des villes.

Mais, plus largement, de l'histoire de l'art à la rénovation urbaine via le patrimoine, la notion de paysage urbain joue de longue date un rôle important pour les acteurs impliqués dans la destruction, conservation ou rénovation des centres villes. Dans cette acception, elle ne provient pas uniquement du milieu professionnel des architectes et urbanistes, mais également des historiens d'art. Par exemple en Italie dès les années 1880, la sauvegarde apparaît comme l'une des composantes fondamentales de l'urbanisme en train de se constituer ${ }^{30}$. Opposés aux hygiénistes et aux ingénieurs, les historiens d'art (les cultori d'architettura e d'arte antica) se consacrent à la défense des centres anciens alors touchés par les opérations d'assainissement et d'éventrement. Aussi étendent-ils la notion de conservation du monument isolé à celle d'ensemble urbain : prend alors forme l'idée d'ambiente artistico, soit l'identité historico-artistique du centre ancien fondée sur l'homogénéité stylistique et la variété volumétrique, le terme d'ambiente véhiculant quant à lui les valeurs de milieu et d'environnement, avec une forte connotation historique. Dans cette conception, le paysage urbain désigne un continuum, où les valeurs plastiques, volumétriques et historiques de chaque édifice comptent moins que celles de l'ensemble. Dans les articles des historiens d'art, la locution est équivalente à celle de "visage de la ville", voire à "visage du pays", formes de représentation de l'«identité de la nation ». On peut mettre en parallèle de telles transformations - du monument au paysage - avec celles observées par B. Notteboom dans les actions de la Commission des sites en Belgique, au début du Xx siècle, ainsi que les connotations patriotiques associées dans ce pays au paysage urbain ("le visage aimé de la patrie»). Dans cette acception, forgée dans le creuset de l'esthétique urbaine défendue par exemple par Camillo Sitte ou Charles Buls, la notion de paysage urbain s'inscrit dans les processus d'esthétisation urbaine ${ }^{31}$.

Du patrimoine à la photographie : processus d'esthétisation urbaine

Le paysage urbain dans son acception patrimoniale s'est ensuite diffusé dans les discours professionnels puis dans les discours critiques. Dans les années 1960, il ne recouvre plus uniquement des valeurs esthétiques ou patrimoniales, mais également sociales. Il est alors associé à une démarche de conservation qui outre les caractères architecturaux, artistiques de la ville, envisage le maintien sur place de sa population, même démunie ou fragile. Illustrée dans ce volume par l'essai d'Italo Zannier, la rénovation du centre historique de Bologne, opération conduite par l'urbaniste en chef Pierluigi Cervellati et l'historien d'art Andrea Emiliani, a constitué en 1970 un projet emblématique de cette démarche, s'inscrivant dans la revendication politique et sociale de nouveaux modèles de développement pour les villes. Considérée comme exemplaire, cette opération qui a connu de nombreux échos positifs hors des frontières italiennes a retenu notre attention, car elle articule en outre les enjeux de patrimoine et ceux d'esthétisation du paysage urbain par la photographie. À ce titre, nous présentons dans ce volume quelques prises de vue du photographe italien Paolo Monti, extraites d'un ensemble de 5000 clichés de relevé des rues de Bologne. Mais, processus 
d'esthétisation, la photographie participe ici, selon Pierluigi Cervellati ${ }^{32}$, des instruments opérationnels du projet, au même titre que le relevé ou les archives municipales ayant permis de reconstituer les typologies de l'« architecture mineure » du centre ville, autre notion alors associée au paysage urbain.

Circulation internationale

Partant des recherches de F. Pousin sur le townscape britannique et les échanges avec les États-Unis, nous avions dans le séminaire émis l'hypothèse qu'à travers la circulation internationale de la notion se révèle son épaisseur sémantique (du townscape britannique, au cityscape ou urban landscape américain ${ }^{33}$, enfin aux manifestations de la notion en France, en Italie, et en Allemagne $\left.{ }^{34}\right)$. Les interventions de plusieurs conférenciers ont permis d'amorcer ce volet de la recherche, de commencer à repérer les équivalents de cette notion dans d'autres contextes culturels, enfin d'identifier les décalages temporels avec lesquels elle se diffuse et leur signification, ses altérations sémantiques lorsqu'elle vient s'inscrire dans un nouveau contexte culturel et professionnel. D'un point de vue méthodologique, comme pour toute étude ayant trait à la réception dans des contextes historiques et culturels différents, se posent les problèmes de la traductibilité des termes, de l'équivalence des notions qu'ils recouvrent, enfin, des décalages temporels non négligeables de la diffusion.

De cette analyse comparée, piste pour le moment partiellement exploitée, on peut attendre des résultats quant à la définition et la clarification de la notion elle-même. D'ores et déjà, les investigations sur la circulation ont mis au jour les importations de stratégies éditoriales et visuelles en France, par les paysagistes, à partir des modalités de publication du townscape britannique ${ }^{35}$. Autre résultat, les explorations menées dans plusieurs contextes ont permis de comprendre que la diffusion du townscape réactive au milieu des années 1950 en Italie, presque une décennie plus tard en France, les enjeux de la notion de paysage urbain qui dans chacun de ces pays lui préexiste ${ }^{36}$ avec des significations différentes. D'un point de vue épistémologique et historique, ce résultat n'est pas sans importance.

Le paysage urbain à l'épreuve des transformations de Montréal et Barcelone

Enfin, l'étude de la circulation ne présente pas des intérêts uniquement au niveau historique et épistémologique. La notion de paysage urbain vient croiser des terrains actuels ou historiques et se manifeste dans les pratiques de l'aménagement aujourd'hui, touchant les transformations du paysage des villes, dans des contextes distincts. Deux analyses de transformation urbaine, à des périodes historiques différentes, interrogent le rôle joué par le paysage urbain, à partir de plusieurs enjeux qui ont pu être dégagés tout au long de notre réflexion.

Étudiant la transformation de Montréal en capitale internationale dans les années 1960, André Lortie soulève la question de l'adhésion des acteurs et des habitants aux nouvelles valeurs urbaines portées par les bouleversements que connaît la ville. Une telle question, déjà posée théoriquement dans les années 1950 par les architectes anglais Peter et Alison Smithson (urban reidentification), est ici instruite par une étude précise des représentations à l'œuvre, notamment telles qu'elles sont véhiculées par la photographie ou la littérature qui traduisent un « changement des regards » portés sur Montréal. À l'optimisme des années 1960 pour les grandes transformations succède une inquiétude lisible, notamment dans certaines œuvres photographiques. Celles-ci témoignent de la redécouverte de la ville populaire dont des pans entiers commencent d'être rasés : est à nouveau en question la rénovation urbaine. Le paysage urbain est ici 
au carrefour entre l'histoire des représentations littéraires, photographiques, les pratiques d'aménagement et la patrimonialisation des centres anciens. Tant dans les photographies de Montréal, que celles de Paolo Monti à Bologne, où même celles du paysage flamand dans le Bulletin du Touring Club de Belgique, la photographie représente le mode de saisie d'un paysage urbain en train de disparaître ou menacé de disparition. La perspective de la rénovation/réhabilitation de ces centres a permis de poser une dernière série de questions, à partir d'un autre terrain, plus contemporain. Hovig Ter Minassian fait l'hypothèse de l'appréhension des transformations de Barcelone en termes de paysage urbain, pour ce qu'elle lui permet d'ouvrir des perspectives originales dans la compréhension des phénomènes de gentrification caractérisant la rénovation du centre historique depuis les années 1980 . Son article soulève de forts enjeux méthodologiques et son analyse s'appuie sur une enquête photographique qui vient relayer les nombreux questionnements autour de la photographie présents dans ce volume.

Une telle présence de l'objet photographique dans les contributions ici rassemblées, souligne, s'il en était besoin, le lien privilégié que nous avons pointé à plusieurs reprises, entre cette pratique et le paysage urbain.

\section{NOTES}

1. Voir T. Paquot, «Le paysage urbain, l'écoumène de la modernité ", in C. Younès (dir.), Ville contre nature, philosophie et architecture, Paris, La Découverte, 1999, p. 154-174. 2. Voir les récentes manifestations photographiques telles que " PhotoEspana 2005 » (H. Fernandez commissaire général), Ciudad, PhotoEspana 05 (exhibition book), Madrid, La Fabrica, 2005, 251 p., ou « Paysages d'entre-villes » (N. Chabert et F. Quintin commissaires), Musée Zadkine, catalogue Paris Musées, 2001, 48 p. On évoquera, entre autres, la série « Interrupted City », réalisée par Gabriele Basilico en 1995 et 1996 à Milan, à la demande de la municipalité, G. Basilico, Interrupted City, Barcelona, Actar Edition, 1999, $132 \mathrm{p}$.

3. F. Pousin a été chercheur invité du Centre canadien d'architecture de Montréal en 2000 sur le programme de recherche intitulé « La notion de paysage urbain dans les projets d'architecture et d'urbanisme », voir également F. Pousin, « La création du paysage au risque de l'urbain ", Les annales de la recherche urbaine, $n^{\circ} 85,1999$, p. 32-42, et « Notions et outils pour appréhender le paysage urbain. Une approche épistémologique ", Cahiers de la Méditerrannée, $\mathrm{n}^{\circ} 60$, Paysages urbains ( $\mathrm{xIX}^{\mathrm{e}}-\mathrm{xx}^{\mathrm{e}}$ siècles), t. II, 2000, p. 1-20.

4. Dans cette seconde catégorie, on notera les publications récentes du Centre d'études sur les réseaux, les transports, l'urbanisme et les constructions publiques (CERTU) : A. Prevel, Paysage urbain, à la recherche d'une identité des lieux, Ministère des Transports, de l'Équipement, du Tourisme et de la Mer, Lyon, CERTU, 2006, 159 p. et id., Paysage urbain, un axe d'études à investir?, Ministère des Transports, de l'Équipement, du Tourisme et de la Mer, Lyon, CERTU, 2006, 173 p., qui repartant brièvement des 
définitions du paysage, fait le point sur la demande sociale de paysage en milieu urbain, sur la situation du paysage urbain dans les outils législatifs, les documents d'urbanisme et pour les acteurs de l'aménagement.

5. Pour des raisons éditoriales, nous n'avons pu retenir que les conférenciers des deux premières années du séminaire (2003-2004 et 2004-2005). Nous remercions, pour leur contribution à l'avancée de la réflexion, outre les participants au séminaire, les intervenants de la $3^{\mathrm{e}}$ année, B. Castiglioni (Université de Padoue), V. Ferrario (Institut universitaire d'architecture de Venise), P. Mantziaras (École nationale supérieure d'architecture de Paris-Malaquais), C. Orillard (Université de Paris-VIII), S. Savary (Université de Grenoble).

6. P. Poullaouec-Gonidec, G. Dalmon, S. Paquette (dir.), Paysage en perspectives, Montréal, Presses de l'Université de Montréal, 2005, 358 p.

7. Ibid., p. 303.

8. M. Spens, Paysages contemporains, Paris, Phaïdon, 2005, 239 p.

9. B. Lassus, L. Halprin, M. Swartz, J. Osty, T. Mitani.

10. D. Laroque, B. Saint-Girons (dir.), Paysage et ornement, Paris, Verdier, 2005, 211 p.

11. A. Léon-Miehe, « Le paysage, entre fiction et description », in D. Laroque, B. SaintGirons (dir.), op. cit., p. 17-23.

12. M. Desportes, Paysage en mouvement : transports et perception de l'espace, $\mathrm{XVIII}^{\mathrm{e}}-\mathrm{XX} \mathrm{e}^{\mathrm{e}}$ siècles, Paris, Gallimard, 2005, 404 p.

13. P. Versteegh (dir.), Méandres. Penser le paysage urbain, Genève, Presses polytechniques universitaires romandes, 2005, $192 \mathrm{p}$.

14. F. Chenet-Faugeras, "L'invention du paysage urbain », Romantisme, revue du XIX siècle, n 83, CDU SEDES, 1994, p. 27-37.

15. Elle rassemble les résultats de deux colloques, tenus respectivement en novembre 2002 à Bordeaux et en octobre 2003 à Salzbourg : G. Peylet, P. Kuon, « Paysages urbains de 1830 à nos jours », Eidôlon. Cahiers du Laboratoire pluridisciplinaire de recherches sur l'imaginaire appliqués à la littérature, n 68, mars 2005.

16. Ici illustrées par l'exemple de Bordeaux: M. Saboya, « Aux marges de l'haussmannisation à Bordeaux : le conflit entre histoire et modernité », Eidôlon, cit., p. 85-94 ; id., « Mériadeck, odyssée de l'espace », Eidôlon, cit., p. 241-256. Ces deux essais sur le « paysage réel » entrent malheureusement peu en dialogue avec le reste du volume.

17. G. Peylet, P. Kuon, Eidôlon, cit., p. 9-10.

18. G. Peylet, P. Kuon, Eidôlon, cit., p. 10.

19. F. Chenet, Le paysage et ses grilles (colloque de Cerisy, 1992), Paris, L'Harmattan, coll. «Esthétiques », 1996.

20. Voir F. Pousin, « Construire les visualisations du paysage urbain. Pratiques anglaises et américaines de l'après seconde guerre », Les cahiers de la recherche architecturale et urbaine, $\mathrm{n}^{\circ} 8$, mai 2001, p. 51-61; «Visuality as politics. The example of urban landscape », in M. Dorrian, G. Rose (eds), Deterritorialisation... Revisioning landscape and politics, London, Black Dog Publishing, 2003, p. 161-174 ; « Le paysage urbain, la charge historique d'une notion ", in M. Boisvert (dir.) (avec la coll. de P. Negron-Poblete), L'urbain, un enjeu environnemental, Montréal, Presses de l'Université de Montréal, 2004, p. 217-225.

21. Pour une approche synthétique voir W.M. Whistler, D. Reed, Townscape as a Philosophy of Urban Design, Urban Design Faculty of Architecture and Townplanning, Oxford Polytechnic, 1977. 
22. T. Sharp, Oxford Replanned, London, Architectural Press, 1948.

23. I. de Wolfe (H. de Cronin Hastings), «Townscape ", The Architectural Review, vol. 106, n 636, 1949, p. 354-362 ; G. Cullen, « Townscape Casebook », ibid., p. 363-374.

24. G. Cullen (with R. Matthews), Four Circuit Linear Towns, Leicester and London, De Montfort Press, 1965.

25. J.-M. Schaeffer, Adieu à l'esthétique, Paris, Collège international de philosophie-PUF, 2000, $74 \mathrm{p}$.

26. J. P. Boutinet, « À propos du projet de paysage. Repères anthropologiques », Les carnets du paysage, $\mathrm{n}^{\circ} 7,2001, \mathrm{p} .64-83$.

27. Une opération de recherche sur l'aménagement paysagiste dans les villes nouvelles croisait notre thématique : voir N. Eleb-Harlé, S. Barles et al., « Hydrologie et paysages urbains en villes nouvelles. Morphologie et logiques de conception ", Ministère de l'Équipement, des Transports, du Logement, du Tourisme et de la Mer, Programme d'histoire et d'évaluation des villes nouvelles, octobre 2005, 2 vol. Voir sur les villes nouvelles : V. Claude, J.-C. Fredenucci, « Expériences professionnelles et effets de génération en villes nouvelles (1965-2002) », Ministère de l'Équipement, des Transports, du Logement, du Tourisme et de la Mer, Programme d'histoire et d'évaluation des villes nouvelle, LATTS-ENPC, mai 2004, 2 vol. ; V. Claude, « Les équipes d'aménagement des villes nouvelles. Avatars d'une expérience collective nationale », Les annales de la recherche urbaine, $\mathrm{n}^{\circ}$ 98, octobre 2005, p. 15-24

28. «Le paysage urbain : approches disciplinaires comparées », journée d'étude organisée par le LADYSS et le Laboratoire de géographie sociale et d'études urbaines, EHESS, avec le concours du Centre Maurice Halbwachs, 24 mars 2006. Nous remercions ici particulièrement J. Brun (LADYSS) et M.-V. Ozouf-Marignier (EHESS) qui ont sollicité et coorganisé cette journée, ainsi que les participants D. Nordman (CNRS, CRH et CHSIM), C. Orillard (Université de Paris VIII), S. Valognes (Université de Caen) et M. Volait (CNRS-Citeres).

29. Témoin privilégié, la revue Urbanisme n'est pas le seul vecteur de ces échanges : on consultera, notamment, les ouvrages du Centre de recherche d'urbanisme (CRU). 30. G. Zucconi, La Città contesa. Dagli ingegneri sanitari agli urbanisti, 1885-1942, Milan, Jaca Books, 1989, p. 95-131.

31. À ce propos, on consultera la thèse de doctorat en philosophie d'Y. Belmont, «Esthétique des sites. Architecture du paysage urbain », sous la direction de B. Deloche, Lyon, Université de Lyon-III, 1996, thèse qu'il est venu présenter dans le séminaire, ce dont nous le remercions.

32. P. L. Cervellati, R. Scannavini, C. De Angelis, La nouvelle culture urbaine: Bologne face à son patrimoine, Paris, Seuil, 1981, p. 85-91.

33. Cet aspect a été développé par C. Orillard dans sa contribution au séminaire, en décembre 2005 : « La notion paysage urbain chez Kevin Lynch ».

34. La notion de Stadtlandschaft (littéralement « paysage de la ville » en allemand, mais dont la signification est plus proche de " ville-paysage ») désigne une conception urbaine qui envisage l'interpénétration de la ville et de la nature. Nous devons à la contribution de P. Mantziaras un éclaircissement historique et épistémologique de cette notion chez les architectes et urbanistes allemands : «La ville-paysage : Rudolf Schwarz et la dissolution des villes ", décembre 2005.

35. Voir l'article de F. Pousin dans ce volume.

36. Le townscape a rencontré un ample écho, ce dont attestent les numéros de Architettura cronache e storia à partir de sa fondation en 1955, mais également les 
congrès de l'Istituto nazionale urbanisti (INU) de 1957 et 1959 qui témoignent de l'importance des enjeux professionnels de cette notion, à nouveau articulée au patrimoine et à la défense des sites et des centres villes. Cette recherche, engagée sur l'Italie par H. Jannière, donnera lieu par la suite à des développements.

\section{AUTEURS}

\section{HÉLÈNE JANNIÈRE}

Ladyss (Laboratoire dynamiques sociales et recomposition des espaces), CNRS, 2, rue Valette, 75005 - Paris, janniere@univ-paris1.fr

\section{FRÉDÉRIC POUSIN}

Ladyss (Laboratoire dynamiques sociales et recomposition des espaces), CNRS, 2, rue Valette, 75005 - Paris, frpousin@univ-paris1.fr 